\title{
Enzyme free sensor based on affinity viscosimetry for detection of glucose
}

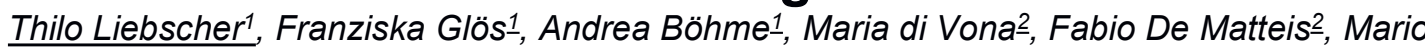

Birkholz프, Andreas Foitzik 1

1 Technical University Wildau, Hochschulring 1, 15745 Wildau,

2 Universita degli Studi di Roma, Faculta Ingenieria Industriale, Via Orazio Raimondo, 18 - 00173 Roma

3 IHP GmbH, Im Technologiepark 25, 15236 Frankfurt (Oder)

liebscher@th-wildau.de

\begin{abstract}
:
The growing demand of miniaturization of cell cultivation new approaches towards measuring and sensing bio-analytes need to be addressed to overcome the challenge of small volumes (less than $150 \mu \mathrm{l})$ containing small amounts of analytes. Most of the available glucose sensors monitor the glucose concentration with the help of enzymes, which become very unstable in terms of long time measurement and consume glucose during the measurement becoming not available anymore for the cells. Therefore, the focus was set on applying an enzyme-free glucose sensor based on a microelectromechanical system (MEMS).
\end{abstract}

Key words: glucose sensing, MEMS, cantilever

\section{Introduction}

Glucose is one of the main molecules, which cells need to survive and grow. It is essential to provide energy from fermentation, aerobic respiration or anaerobic respiration for chemical reactions. Therefore, it is a key factor to be monitored and controlled for any kind of cell growth or cell regulation reactions. One major field of research is cellular research where glucose concentrations need to be constantly monitored. It is carried out mostly inside a bioreaction chamber, which provides constant media flow, controlled environment parameters like temperature or $\mathrm{CO} 2$ concentrations and sensors for verifying the reproducibility of results. A major advantage of these miniaturised reaction chambers are the ability to perform many different experiments at the same time with a reduced amount of space needed [1]. Over the last decade, these reaction chambers have been miniaturised to a degree, that new miniaturised sensors need to be developed. Hereby a major challenge poses the detection of glucose in those small spaces due to two factors. One focus lies on the required size of the sensing apparatus to detect glucose molecules. The other focus lies on the detection of such a small amount of glucose without interfering with the cells consuming glucose to produce energy. Most commonly used glucose sensors are based on an enzymatic reaction to detect glucose, which ultimately converts glucose into another product [2]. The glucose is not anymore available to the cells and therefore cell growth is influenced due to a forced lack of glucose molecules in the media.

\section{Enzyme free detection by BioMEMS}

Different approaches have been investigated to overcome the challenge of enzymes converting glucose into other products. One promising approach represents the use of bio microelectromechanical systems (MEMS) to detect glucose concentrations. First of all a sensoric fluid can be used consisting of dextran and Concanavalin A (Con A). Con A consists of a saccharide-binding site, which at physiological $\mathrm{pH}$-values cross-links between macromolecules of the dextran to form a highly viscous gel phase. If free glucose molecules are present, they are in competition with dextran molecules leading to a change in viscosity (n) of the sensoric fluid, which is shown schematically in Fig. 1 [3]. 

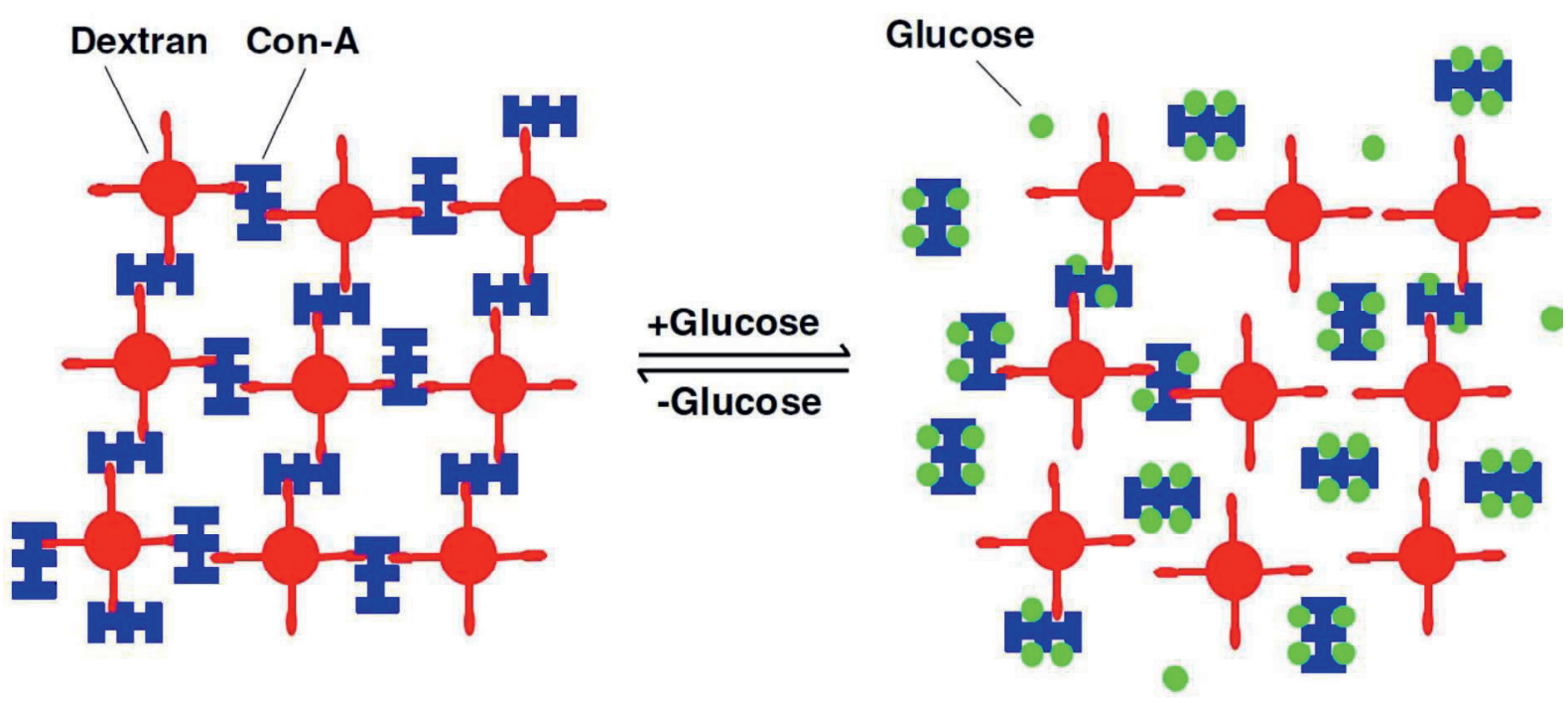

Figure 1: Concanavalin A and dextran mixture without glucose on the left and with free glucose on the right; free glucose molecules are in competition to glucose end-groups of dextran at the Con A binding site leading to a breakup of former Con A / dextran bonds resulting in a decrease of viscosity of the solution [4]

The change in viscosity is detected with a beam, the so-called cantilever, which can be charged electrically. Based on its electric charge the beam is attracted to the ground plate moving through the sensoric fluid. Depending on the viscosity of the sensoric fluid, the beam requires different time intervals to travel from start to end position. This directly correlates to the concentration of free glucose molecules in the solution or surrounding fluid. The basic concept for this method is shown in Fig 2.

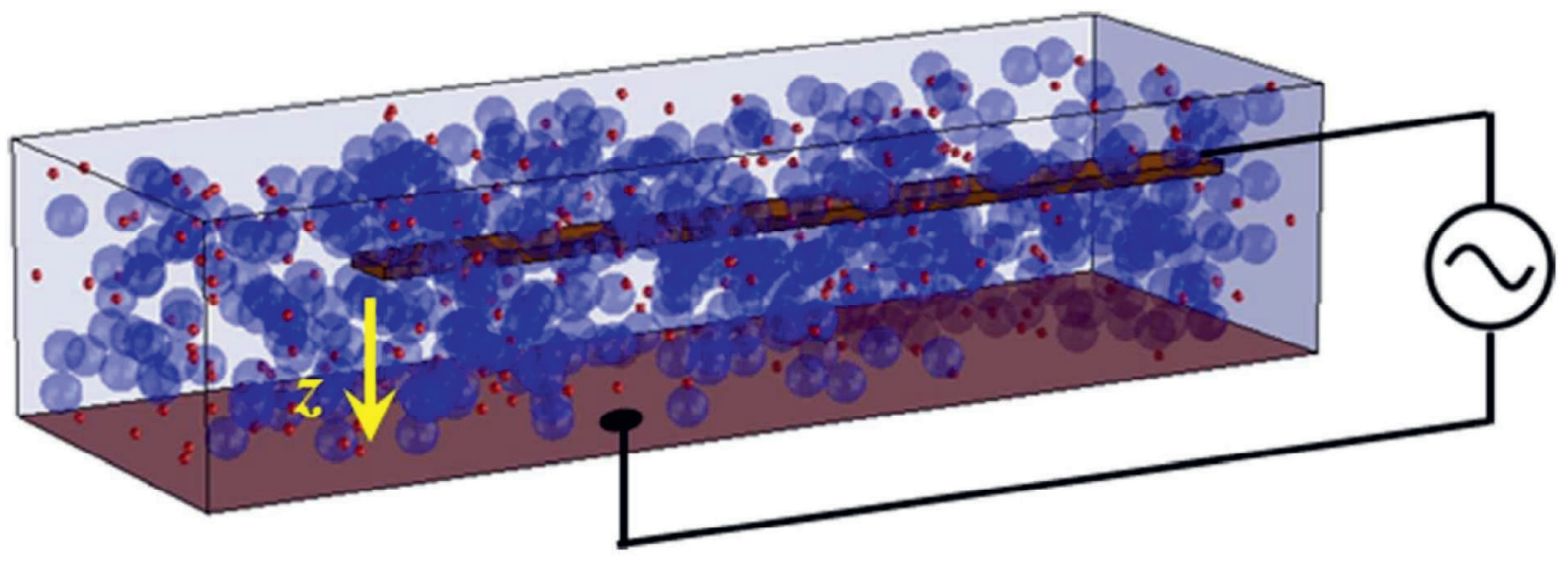

Figure 2: sensoric fluid is represented by circles (red and blue); a beam is placed inside the sensoric fluid and can be electrically charged; travel time of the beam through the fluid can be detected [5]

This sensing method will be applied in a microbiological environment. All materials are required, to be stable against bio-corrosion and are able to be charged electrically when needed. Titanium-nitride represents one of the possible materials, which can also be prepared with CMOS applications [6]. The designed chip layout is shown in Fig. 3 representing an Xshaped beam for viscosity detection. The chip is placed inside a silicon body, which possesses a cavity for the sensoric fluid. A membrane separates the sensoric fluid from the media letting only molecules pass with up to $6 \mathrm{kDa}$ in weight. 


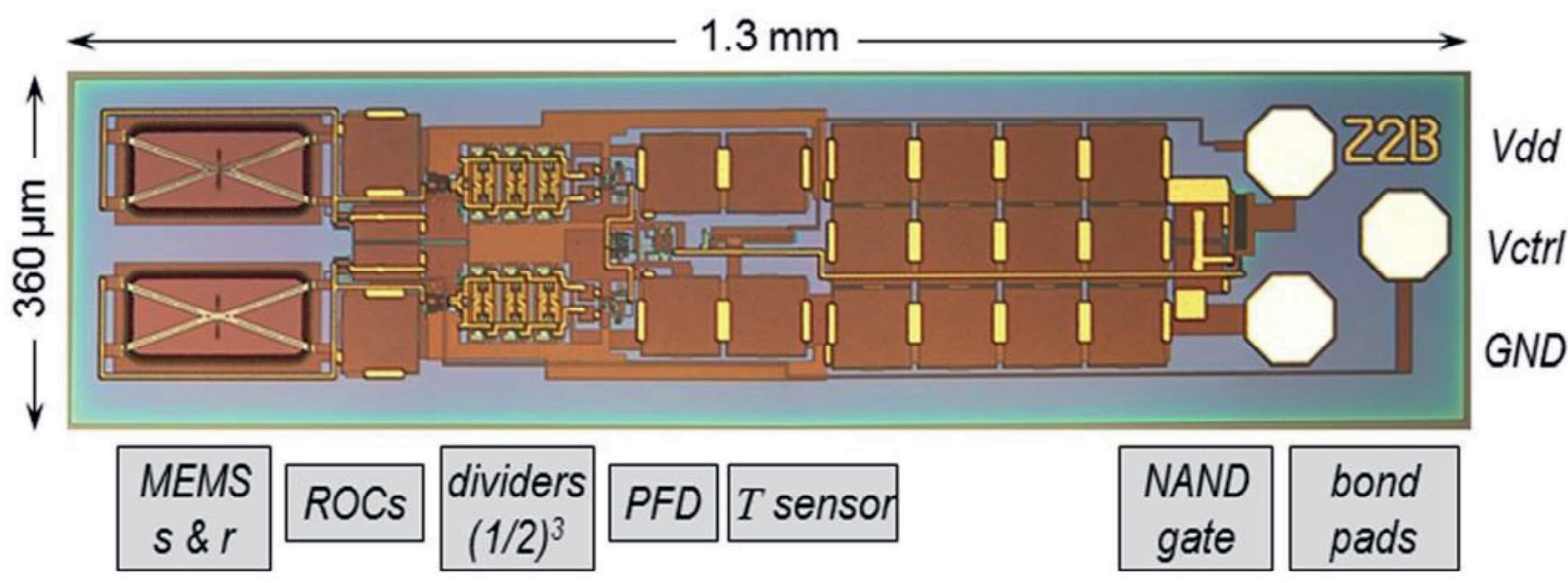

Figure 3: design of the chip used for detection of changes in viscosity; design shows an example of an X-shaped beam for the sensor [5]

The transformation cascade of the signal is reflected by the change in glucose concentration resulting in a shift in viscosity. This is followed by the change of the beam deflection time leading to an increase or decrease in capacitance and frequency tuning. A defined voltage, a so-called $\mathrm{V}$-control, is set and the beam switch-time $t_{s w}$ is detected. Afterwards it can be correlated to the glucose concentration. V-control measurements have been performed using a micro-bioreaction chamber with X-shaped beam MEMS used inside the sensor chamber.

\section{Defining V-control values}

For the detection of different glucose concentrations, a voltage is required, which induces a beam switch with a low Noise-toSignal ratio during the measurement. A setup a glucose concentration of $2.25 \mathrm{mg} / \mathrm{ml}$ at $37^{\circ} \mathrm{C}$ stirred was used to detect the best voltage
DMEM media over 12 hours. The sensor chip was placed in a micro dialyser tube with a $6 \mathrm{kDa}$ membrane cut off. Furthermore, a workstation is required to record data and a software, in this case MibsView, to set different voltage levels. Based on the manufactures guidance on how the chip was produced a voltage, a control voltage (V-control) range from $35 \%$ to $70 \%$ of $V_{d d}$ is set over the given time. The basic steps for analysing a given $\mathrm{V}$-control are
1. set $\mathrm{V}$-control
2. start analysis
3. detect switch time every 10 seconds over 20 minutes.

These steps are repeated for every pre-defined $\mathrm{V}$-control setting until the maximum $\mathrm{V}$-control value has been reached. Fig. 4 shows the graph received from the analysis of the V-control.

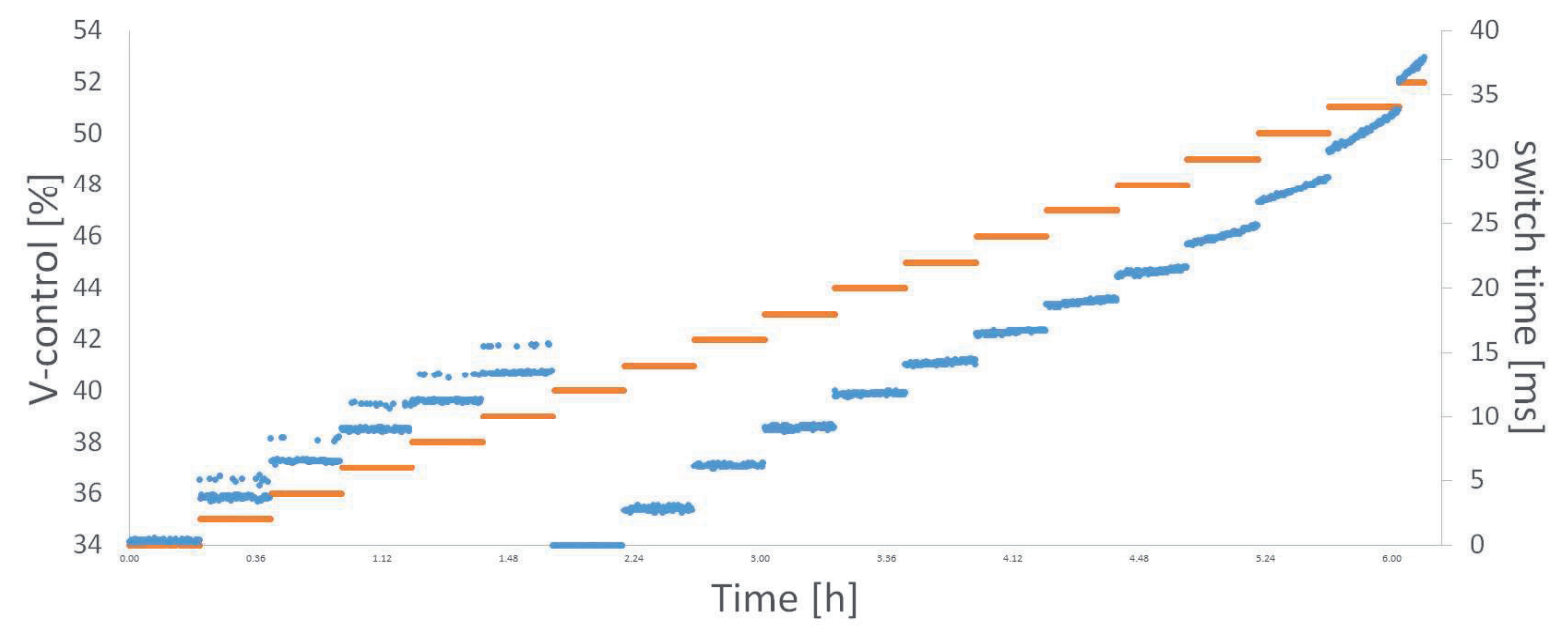

Figure 4: set $V$-control (orange), detected switch time for set $V$-control (blue), switch time below $V$ control $40 \%$ are uneven whereas $V$-control above $40 \%$ are smooth till $V$-control of $47 \%$, low spread of measured switch times indicate a low Noise-to-Signal ratio required for glucose concentration analysis 


\section{Results for V-control values}

For this measurement, the $\mathrm{V}$-control range was set to be between $34 \%$ and $52 \%$. The $\mathrm{V}$-control range is defined by the manufacture's processing of the chip. Every 20 minutes the $\mathrm{V}$ control was increased by one and the occurring switch time detected. It is important to increase the $\mathrm{V}$-control only in small steps to detect the lowest Noise-to-Signal ratio to reduce any errors during the glucose concentration analysis later on. First, the raw detection data has to be enhanced and modified to calculate the Noiseto-Signal ratio. Therefore, faulty data have to be removed, which contain switch times below $0.5 \mathrm{~ms}$. Afterwards the Noise-to-Signal ratio can be calculated with the following equation:

$$
N S R=\frac{P_{\text {noise }}}{P_{\text {signal }}}=\frac{\sigma}{\mu}
$$

Hereby is $\sigma$ the standard deviation of the noise and $\mu$ the mean of the signal. Table 1 shows the detected switch times and their corresponding calculated data.

Table 1: V-control values with the mean switch time and standard deviation as well as their corresponding Noise-to-Signal ratio, $V$-control of $40 \%$ represents a measurement error due to switch time below $0,5 \mathrm{~ms}$

\begin{tabular}{|c|c|c|}
\hline V-control & $\begin{array}{c}\text { Mean switch } \\
\text { time } \mu[\mathrm{ms}]\end{array}$ & $\begin{array}{c}\text { Noise-to- } \\
\text { Signal ratio }\end{array}$ \\
\hline$<40$ & 7,61 & 0,11 \\
\hline 40 & 0,02 & 0,12 \\
\hline 41 & 2,81 & 0,05 \\
\hline 42 & 6,21 & 0,02 \\
\hline 43 & 9,14 & 0,01 \\
\hline 44 & 11,80 & 0,0081 \\
\hline 45 & 14,24 & 0,009 \\
\hline 46 & 16,60 & 0,008 \\
\hline 47 & 18,94 & 0,01 \\
\hline 48 & 21,35 & 0,0081 \\
\hline$>48$ & $>24,1$ & $>0,017$ \\
\hline
\end{tabular}

As shown in Table $1 \mathrm{~V}$-control set below $40 \%$ or above $48 \%$ correspondents to high Noise-toSignal ratios, which are not usable for any glucose concentration analysis. The best Noiseto-Signal ratio was detected at a $\mathrm{V}$-control of $46 \%$ with 0.008 . The corresponding switch time averages at around $16.6 \mathrm{~ms}$ per switch, which is optimal for the beam since it is not too slow or too fast, therefore could result in breakage of the beam. The N-population for given results is sufficient to show a statically significant Noiseto-Signal ratio. Fig 5 . shows the different $\mathrm{V}$ controls of the lowest Noise-to-Signal ratios demonstrating a V-control of $46 \%$ as optimal value.

Taking a closer look at the values of other $\mathrm{V}$ controls shows that $V$-control of $44 \%$ or $48 \%$ are in close range to the best Noise-to-Signal ratio, but their switch time is too short respectively too long for a glucose concentration at $2.25 \mathrm{mg} / \mathrm{ml}$. A mean switch time of $16.6 \mathrm{~ms}$ represents an optimal switch time in relation to a small standard deviation. It is necessary to obtain a sufficient signal value for reproducible experiments with known and unknown glucose concentration. A V-control lower than $46 \%$ with its corresponding switch time of $14.24 \mathrm{~ms}$ or lower represents a signal strength, which is not satisfactory for further tests with the sensor. Lower V-control values show even faster switch times and therefore cannot be considered for further testing. In addition, the corresponding Noise-to-Signal ratios are above the optimal ratio, which leads to the exclusion of these values. Opposite to low $\mathrm{V}$-control values, high $\mathrm{V}$-control values of $47 \%$ or above show a satisfactory switch time.

On the downside, these switch time signals are paired with a high standard deviation marking a high variation in those values. This corresponds to the Noise-to-Signal ratio indicating that during measurement of known or unknown glucose concentrations errors will influence the analysis. Thus, if an unknown glucose concentration is to be analysed its respective switch time tsw could result in an error during the measurement since the $\mathrm{V}$-control switch time was too fast. In addition, very rapid switch times can also induce extremely high stress on the beam followed by irreversible bending or even breakage. Therefore, the switch time must be viewed in context with the used glucose concentration for the measurement.

This glucose sensor will be used mostly in biological environments, which are in the range of $0 \mathrm{mg} / \mathrm{ml}$ to $2.5 \mathrm{mg} / \mathrm{ml}$ of glucose in a solution. As mentioned earlier in this experiment a glucose concentration of $2.25 \mathrm{mg} / \mathrm{ml}$ was used representing a concentration close to the maximum of the glucose concentration range. Thus, the switch time of $16.6 \mathrm{~ms}$ represents a close to the maximum achievable switch time and can be considered an optimal switch time without the risk of bending or breaking the beam. With the received $\mathrm{V}$-control of $46 \%$ being optimal for calibration measurement due to its low Noise-to-Signal ratio and sufficient switch time of $16.6 \mathrm{~ms}$ further steps can be taken to detect unknown glucose concentrations. 


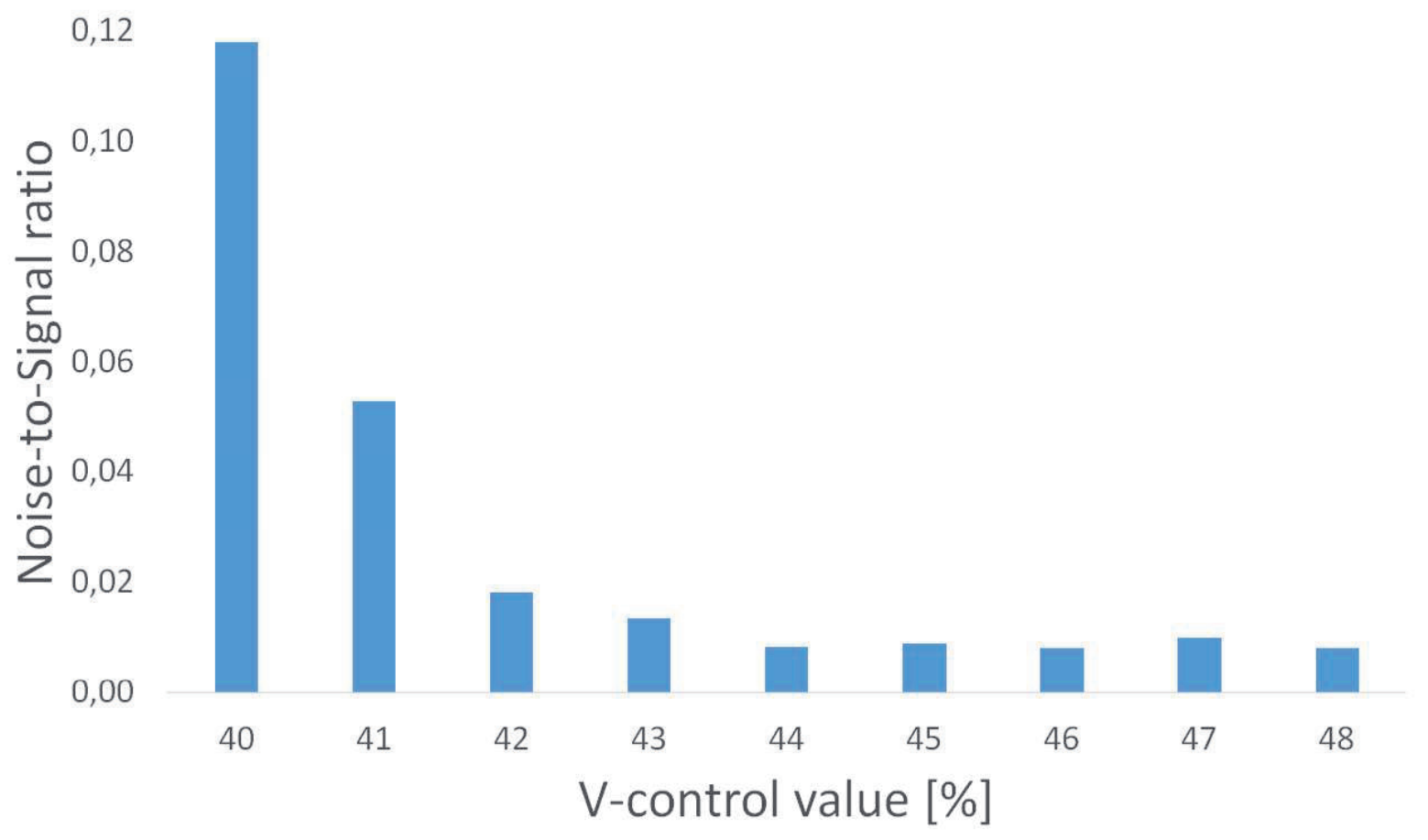

Figure 5: Noise-to-Signal ratio for corresponding V-control values; V-control of $46 \%$ presents the optimal Noise-toSignal ratio with 0.008

\section{Outlook}

Finding the optimal V-control was one key aspect to take the next step in calibrating the sensor with known glucose concentrations. It is necessary to analyse these glucose concentrations with a close to zero Noise-toSignal ratio to reduce any errors in generating the calibration graph. A possible error are $\mathrm{pH}$ drifts during the measurement, which shall be kept constant for further analysis. After achieving a close to error free calibration curve the detection of unknown glucose concentrations can be achieved. This shall be carried out in a micro-bioreaction chamber due to the small size of the sensor.

\section{Acknowledgement}

We acknowledge the help of Lorenz Theuer for writing the Matlab program for V-control analysis. The Company Sitec-Sensortechnik for MIBSview to record switch times. Thomas Voss for flip-chip bonding the sensors and Mario Birkholz for supplying IHP sensor chips. The Company Scienova for supplying the Micro Dialyzer Tubes for analysis.

\section{References}

[1] A. Böhme, G. Gatomski, C. Giese, C.-H. Edel und A.-H. Foitzik, „A disposable and miniaturized bioreactor system for applications in cell and tissue based experimental in in-vitro environments, " Journal of Engineering and Technology, Bd. 1, 2010.
[2] J. Raba und H. A. Mottola, „Glucose oxidase as an analytical reagent," Critical reviews in Analytical chemistry, Bd. 25, Nr. 1, pp. 1-42, 1995.

[3] K. D. Hardman und C. F. Ainsworth, „Structure of concanavalin A at 2.4-Ang resolution," Biochemistry, Bd. 11, Nr. 26, pp. 4910-4919, 1972.

[4] Y. Zhao, S. Li, A. Davidson, B. Yang, Q. Wang und $Q$. Lin, „A MEMS viscometric sensor for continuous glucose monitoring," Journal of micromechanics and microengineering, Bd. 17, Nr. 12, p. 2528, 2007.

[5] M. Birkholz, K.-E. Ehwald, T. Basmer, P. Kulse, C. Reich, J. Drews, D. Genschow, U. Haak, S. Marschmeyer, E. Matthus und others, „Sensing glucose concentrations at $\mathrm{GHz}$ frequencies with a fully embedded Biomicro-electromechanical system (BioMEMS)," Journal of Applied Physics, Bd. 113, Nr. 24, pp. 244904-244904, 2013.

[6] M. Birkholz, K.-E. Ehwald, D. Wolansky, I. Costina, C. Baristiran-Kaynak, M. Frohlich, H. Beyer, A. Kapp, and F. Lisdat, Surf. Coat. Technol. 204, 2055 (2010). 